\title{
MICROWAVE MEASUREMENT OF COAL LAYER THICKNESS
}

Doyle A. Ellerbruch

John W. Adams

Electromagnetics Division

Institute for Basic Standards

National Bureau of Standards

Boulder, Colorado 80302

September 1974

Final Report

Prepared for

U. S. Bureau of Mines

United States Department of the Interior

Pittsburgh, Pennsylvania 15222

Working Fund Agreement SO 144086 


\section{NBSIR 74-387}

\section{MICROWAVE MEASUREMENT OF COAL LAYER THICKNESS}

Doyle A. Ellerbruch

John W. Adams

Electromagnetics Division

Institute for Basic Standards

National Bureau of Standards

Boulder, Colorado 80302

The views and conclusions contained in this document should not be interpreted as necessarily representing the official policies or recommendations of the Interior Department's Bureau of Mines of the U. S. Government.

September 1974

Final Report

Prepared for

U. S. Bureau of Mines

United States Department of the Interior

Pittsburgh, Pennsylvania 15222

Working Fund Agreement SO 144086

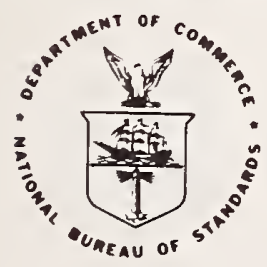

U.S. DEPARTMENT OF COMMERCE, Frederick B. Dent, Secretary NATIONAL BUREAU OF STANDARDS. Richard W Roberts Director 



\section{FOREWORD}

This report was prepared by the National Bureau of Standards, Boulder, Colorado, under USBM Contract No. S0 144086. It was administered under the technical direction of the Pittsburgh Mining and Safety Research Center with Mr. M. Pazuchanics acting as the technical project officer.

This report is a summary of the work completed as part of this contract during the period June 1974 to September 1974. This report was submitted by the authors in October 1974 . 
1.0 INTRODUCTION ....................................

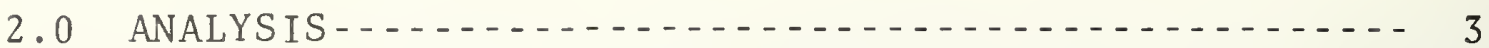

2.1 Properties of a Medium-.............. 3

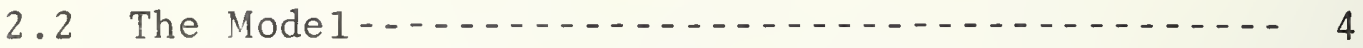

2.3 Dielectric Constant Determination-.......- 7

3.0 EXPERIMENTAL RESULTS -............................ 8

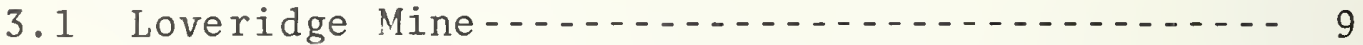

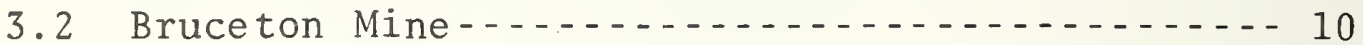

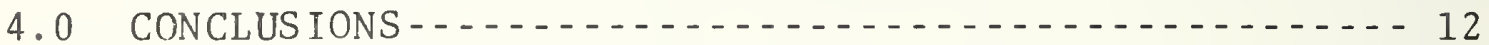

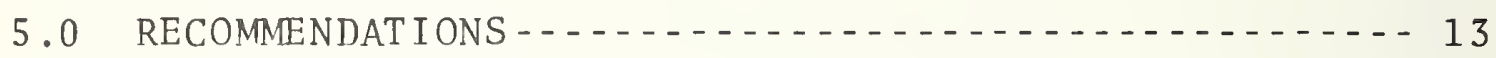

6.0 ACKNOWLEDGMENTS - - - - 14

7.0 REFERENCE - $\ldots \ldots$

APPENDIX - _ _ $\ldots \ldots \ldots$

LIST OF ILLUSTRATIONS

$\underline{\text { Page }}$

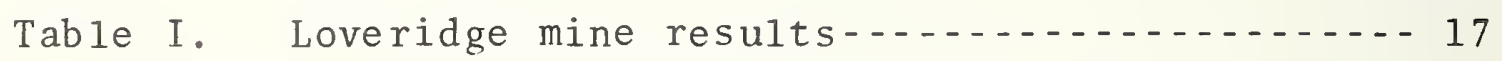

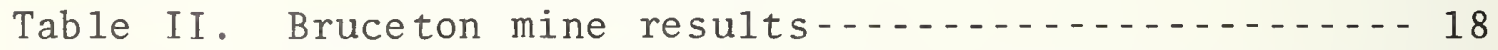

Figure 1. Model used for illustration and calculations-19

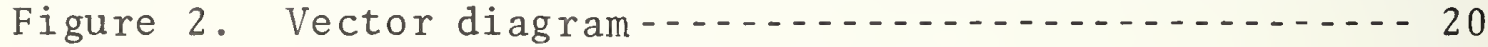

Figure 3. Block diagram of measurement system-....-.-21

Figure 4. Measurement setup inside Bruceton Mine-.-.-2 22

Figure 5. Measured data, $10 \mathrm{~cm}$-thick layer of coal---- 23

Figure 6. Calculated data, $10 \mathrm{~cm}$-thick layer of coal--- 24

Figure 7. Measured data, $20 \mathrm{~cm}$-thick layer of coal---- 25

Figure 8. Calculated data, $20 \mathrm{~cm}$-thick layer of coal--- 26

Figure 9. Measured data, $40 \mathrm{~cm}$-thick layer of coal---- 27

Figure 10. Calculated data, $40 \mathrm{~cm}$-thick layer of coal--- 28

Figure 11. Photo of 40-cm thick layer of coal-.-.-.--29 
The possibility of using a microwave system to measure coal layer thickness in a mine was investigated. Measurements were made in two different mines near Pittsburgh, $\mathrm{Pa}$., and near Fairview, West Virginia. Frequencies in the range $0.5 \mathrm{GHz}-4.0 \mathrm{GHz}$ were used to measure samples between $10-40 \mathrm{~cm}$ thick. All samples were backed with a naturallyoccurring draw slate.

The results indicated a definite possibility of determining layer thickness in most cases, although anomalies may introduce enough error to give misleading results in some cases. Anomaly detection may be very useful in some cases. More experimental data and theoretical study are needed for complete verification.

Data from a model and measured data show considerable agreement. The real value of this model is to point out causes and effects, and what additional information is needed in order to obtain a measurement system that is optimum under most conditions.

The dielectric constant of coal is apparently a function of moisture content. Draw slate seems to have a significantly higher dielectric constant than coal. Also, a layer of coal having different electrical characteristics within the layer was detected with the microwave system.

This technique has great potential if fully developed. It would have significant impact in areas of energy, safety, and productivity.

Key words: Automation; coal; coal mine safety; dielectric constant; energy; microwave measurement; nondestructive testing; thickness of coal layer.

\section{$1.0 \quad$ INTRODUCT ION}

The present-day needs for determining the thickness of the coal layer left on mine-haulageway roofs is related primarily to mine safety. A layer of sufficient thickness is considered necessary for roof-strength purposes, and in the case of draw slate (in the Pittsburgh seam), to prevent 
exposure of the slate to air. When exposed to air, draw slate deteriorates very rapidly and greatly increases the chance of a roof fall. In addition to the safety aspect, a measurement system that could non-destructively monitor layer thickness would aid production and possibly allow further automation of control of a continuous miner.

Present mining practice is to stop mining every ten feet to measure coal-layer thickness on the roof by drilling through the coal to the draw slate. The drill operators determine when the slate has been reached as verified by a change in color of the drill chips. The color changes from coal black to dull gray. Holes are drilled at approximately ten-foot intervals along one side of the mine haulageway.

The future need for thickness measurements is related to the need for automated mining machines. A sensor is required to continuously monitor coal-layer thickness and provide a signal for vertical control of the automated machine. It is desirable that the sensor be non-contacting with the coal, and it is necessary that the sensor be permissible, operate remotely, and be able to operate in a very wet and dusty environment.

The application of microwave technology to the roof coal thickness measurement problems presents a possibility of satisfying many present and future needs. Microwave signals are capable of penetrating and interacting with coal in such a way that they do not physically affect the coal, and yet the interaction will provide the necessary information about coal. A sensor which utilizes microwave signals can be constructed to be non-contacting, to provide data continuously for monitoring and control purposes, and to be permissible.

There is exciting potential for a fully-developed system applying this microwave technique through non-destructive, real time measurements. Safety, energy, self sufficiency, automation, and increased productivity all would be impacted. 
This effort consisted of investigating the relationship between coal electromagnetic penetrability, as a function of microwave frequency, and of reflections set up by the drawslate-coal interface. Measurements were made in the Loveridge Mine near Fairview, West Virginia, and in the Bruceton Mine near Pittsburgh.

In all cases the coal samples tested consisted of an undisturbed coal layer backed by a layer of draw slate approximately $40 \mathrm{~cm}$ thick. The draw slate was backed by a layer of "wild" coal (definitive description unknown) of unknown thickness.

\section{$2.0 \quad$ ANALYS IS}

\subsection{Properties of a Medium}

The following equations outline the properties of a complex medium [1]. In general, waves propagating in a medium undergo attenuation and phase shift as they propagate. The complex propagation constant for plane waves is

$$
\gamma=i \omega \sqrt{\mu \varepsilon}\left[1-i \frac{\sigma}{\omega \varepsilon}\right]^{1 / 2}
$$

and can be written as

$$
\gamma=\alpha(\omega)+i \beta(\omega) .
$$

Solving for $\alpha(\omega)$ and $\beta(\omega)$ gives

$$
\begin{aligned}
& \alpha(\omega)=\left\{\frac{\omega^{2} \mu \varepsilon}{2}\left(\sqrt{1+\left(\frac{\sigma}{\omega \varepsilon}\right)^{2}}-1\right\}\right\}^{1 / 2} \\
& \beta(\omega)=\left\{\frac{\omega^{2} \mu \varepsilon}{2}\left(\sqrt{1+\left(\frac{\sigma}{\omega \varepsilon}\right)^{2}}+1\right)\right\}^{1 / 2} .
\end{aligned}
$$

The intrinsic impedance of a medium is given by:

$$
\eta=\sqrt{\frac{\mu}{\varepsilon\left(1-i \frac{\sigma}{\omega \varepsilon}\right)}} .
$$


However, the assumption that $\frac{\sigma}{\omega \varepsilon} \ll 1$ will be made in the following model and analysis, except for calculations of $\alpha(\omega)$.

\subsection{The Mode 1}

The following discussion outlines the mode 1 and the equations used in these measurements.

The model used consists of two layers of finite thickness sandwiched between two semi-infinite half-spaces. In the real world, these layers are air, coal, draw slate, and "wild" coal (see figure 1). The media are assumed to be isotropic and homogeneous with plane boundaries. Electrical properties of each layer are described by permeability $(\mu)$, permittivity $(\varepsilon)$, and conductivity $(\sigma)$. All layers are assumed non-magnetic, i.e., $\mu_{i}=\mu_{0}$, where $\mu_{0}=4 \pi \times 10^{-7} \mathrm{hy} / \mathrm{m}$. Additionally, $\varepsilon$ is assumed to be independent of frequency. Ray paths for electromagnetic energy are also assumed.

Due to the directivity of the ideal horns assumed in the mode1, only reflected waves are received. In practice, considerable design, placement, and polarization strategy are required to reduce the direct coupling to an acceptable level. If horns were close enough to ideal to prevent a direct coupling of energy, lower minima could be detected. Also, the horns are not directive enough to fully justify use of a singleray mode1. Nevertheless, this model is instructive in determining what is important.

For E normal to plane of incidence, impedance, $Z$, from medium o to medium 1 is

$$
\begin{aligned}
& z_{0}=\eta_{0} \sec \theta_{0} \\
& z_{1}=\eta_{1} \sec \theta_{1},
\end{aligned}
$$

and 


$$
\Gamma_{01}=\frac{z_{1}-z_{0}}{z_{1}+z_{0}}=\left(\frac{\frac{\sec \theta_{1}}{\sqrt{\varepsilon_{1}}}-\frac{\sec \theta_{0}}{\sqrt{\varepsilon_{0}^{T}}}}{\frac{\sec \theta_{1}}{\sqrt{\varepsilon_{1}}}+\frac{\sec \theta_{0}}{\sqrt{\varepsilon_{0}}}}\right)=\left(\frac{\cos \theta_{0} \sqrt{\varepsilon_{0}^{T}}-\cos \theta_{1} \sqrt{\varepsilon_{1}^{\prime}}}{\cos \theta_{0} \sqrt{\varepsilon_{0}^{1}}+\cos \theta_{1} \sqrt{\varepsilon_{1}^{1}}}\right) .
$$

$\theta_{1}$ is calculated according to Snell's law as

$$
\theta_{1}=\sin ^{-1}\left(\left(\sin \theta_{0}\right)\left\{\frac{\varepsilon_{0}}{\varepsilon_{1}}\right)\right\} \text {. }
$$

The resultant field from single reflected waves from each of the three boundaries are:

$$
\begin{aligned}
E_{y}= & E_{0} e^{-i 2 \beta_{0} s}\left\{\Gamma_{0}+\Gamma_{1}\left(1-\left|\Gamma_{0}\right|^{2}\right) e^{-2 \alpha_{1} \ell} e^{-i 2 \beta_{1} \ell}\right. \\
& +\Gamma_{2}\left(1-\left|\Gamma_{0}\right|^{2}\right)\left(1-\left|\Gamma_{1}\right|^{2}\right) e^{-2\left(\alpha_{1} \ell+\alpha_{2} d\right)} \times \\
& \left.e^{-i 2\left(\beta_{1} \ell+\beta_{2} d\right)}\right\},
\end{aligned}
$$

where $E_{y}$ is the component of $E$ normal to the page in figure 1. Multiple reflections in layer one can be described by an infinite series of terms:

$$
\sum_{n=1}^{\infty} \Gamma_{1}\left(\Gamma_{0} \Gamma_{1}\right)^{n}\left(1-\left|\Gamma_{0}\right|^{2}\right) e^{-2(n+1) \alpha_{1} l} e^{-i 2 \beta_{2} \ell(n+1)} .
$$

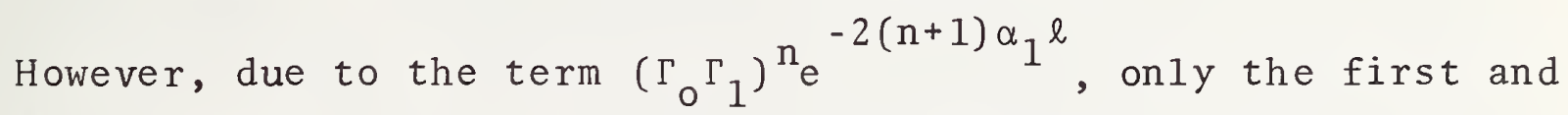
second terms of the series will be necessary in typical coal layer cases. Multiple reflections in layer 2 are neglected. When parameters that determine values of $\beta$ are inserted, along with two reflections in layer one, then $\mathrm{E}_{\mathrm{y}}$ is given by 


$$
\begin{aligned}
& E_{y}=E_{0} e^{-i 2 \beta_{0} s}\left(\Gamma_{0}+\Gamma_{1}\left(1-\left|\Gamma_{0}\right|^{2}\right) e^{-2 \alpha} 1_{e}^{\ell-i \frac{2 \omega l}{c} \sqrt{\varepsilon_{1}^{l}}} \cos \theta_{1}\right. \\
& +\Gamma_{2}\left(1-\left|\Gamma_{0}\right|^{2}\right)\left(1-\left|\Gamma_{1}\right|^{2}\right) e^{-2 \alpha_{1} \ell} e^{-2 \alpha_{2} d} e^{-i \frac{2 \omega \ell}{c} \sqrt{\varepsilon}} \cos \theta_{1} \times \\
& e^{-i \frac{2 \omega d}{c} \sqrt{\varepsilon_{2}^{\prime}}} \cos \theta_{2} \\
& +\Gamma_{1}\left(1-\left|\Gamma_{0}\right|^{2}\right)\left(\Gamma_{0} \Gamma_{1}\right) e^{-4 \alpha} 1^{\ell} e^{-i \frac{4 \omega \ell}{c} \sqrt{\varepsilon} !} \cos \theta_{1} \\
& +\Gamma_{1}\left(1-\left|\Gamma_{0}\right|^{2}\right)\left(\Gamma_{0} \Gamma_{1}\right)^{2} e^{-6 \alpha_{1} \ell} e^{\left.-i \frac{6 \omega \ell}{c} \sqrt{\varepsilon_{1}^{\prime}} \cos \theta_{1}\right)} \text {. }
\end{aligned}
$$

The various $\varepsilon^{\prime}$ denote relative dielectric constants, where $\varepsilon=\varepsilon^{\prime} \varepsilon_{0}$, and $\varepsilon_{0}$ is permittivity of free space, $8.854 \times 10^{-12} \mathrm{fd} / \mathrm{m}$. At least to first order, the spacing between the antennas and the air-coal interface makes no difference in this technique, as its effect is factored to the outside of the bracket (in eq. (10)). Examination of the first and second terms inside the bracket shows how the thickness, l, of layer one is determined. The reflection coefficient, $\Gamma_{0}$, is a stationary vector, while $\Gamma_{1}\left(1-\left|\Gamma_{0}\right|^{2}\right) e^{-2 \alpha} 1^{\ell}$ is a rotating vector as shown in figure 2. The magnitude of the resultant vector will go through successive minima (or maxima) every $2 \pi$ radian rotation of the second vector. In this case, $\omega$ is varied. Thus, solving for $l$ in terms of frequencies of two successive minima in the argument,

gives:

$$
\frac{2 \omega l}{c} \sqrt{\varepsilon_{1}^{\prime}} \cos \theta_{1} \text {, }
$$

$$
l=\frac{c}{2\left(f_{2}-f_{1}\right) \sqrt{\varepsilon_{1}} \cos \theta_{1}} .
$$


where successive minima of the resultant vector occur at $f_{1}$ and $f_{2}$. Argument $\theta_{1}$ is very close to zero for all reasonable angles of incidence, and even for grazing incidence, would be only about $20^{\circ}$. Therefore the most important parameter is $\varepsilon_{i}$. Unfortunately, this is not the only parameter needed, as there are other rotating vectors present also. Their magnitudes may obscure the desired layer thickness if attenuations and reflection coefficients do not reduce their amplitudes sufficiently.

The simplifying assumptions made throughout this analysis are subject to question; no judgment can be made as to the validity of these assumptions without adequate information on properties of materials, antennas, etc. This information can be obtained from extensive measurements made in situ.

\subsection{Dielectric Constant Determination}

Obviously, $\varepsilon^{\prime}$ of coal, as well as slate and other roof materials, must be known with fairly good accuracy. Unfortunately, coal is not isotropic, and its dielectric constant is strongly influenced by the amount of moisture present. Results in the next section show this to be true for the two mines visited, where $\varepsilon^{\prime}$ varied from less than 5 in a relatively dry mine to over 7 in a relatively wet mine. The exact moisture content is not known. The fact that moisture influences the properties of materials has been documented in other cases [2]. Thus, values taken from laboratory measurements are not likely to be suitable in this situation [3].

There are at least three ways of measuring $\varepsilon^{\prime}$ in situ. The first method involves measuring $\Gamma$ at an air-coal interface. From

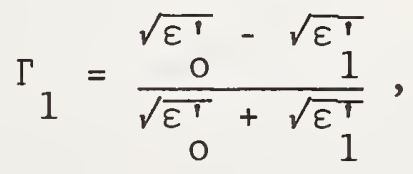


$\varepsilon_{0}^{\prime}=1$, then

$$
\varepsilon_{i}=\left(\frac{1+|\Gamma|}{1-|\Gamma|}\right)^{2} .
$$

This method assumes the medium $\varepsilon_{i}$ is sufficiently lossy to appear as an infinite half space.

A second method is to use eq. (8), and physically measure the coal layer thickness. Then $\varepsilon^{\prime}$ can be determined.

A third method for determining the dielectric constant is to utilize a change in depth of the coal layer where the absolute depths are not known, but the change of depth is known. Assuming a step change in depth, (8) is rewritten as

$$
\ell_{i}^{\prime}=\frac{c}{2 \sqrt{\varepsilon^{\prime} \Delta f^{\prime}}},
$$

where $\Delta f^{\prime}=f_{2}^{\prime}-f_{1}^{\prime}$ and the primed values correspond to another depth. Taking the difference between (11) and (14) and solving for the dielectric constant results in

$$
\varepsilon^{\prime}=\left(\frac{c}{2 \Delta l}\right)^{2}\left(\frac{1}{\Delta f}-\frac{1}{\Delta f^{\prime}}\right)^{2} \text {. }
$$

All three methods for determining the dielectric constant of coal and the draw slate were utilized during these measurements. The results will be given in the next section.

\subsection{EXPERIMENTAL RESULTS}

This measurement technique has been used previously to measure layer thickness of slush hydrogen. This instrumentation is not practical for coal-mine measurements, but was used only to determine feasibility.

Approximately $225 \mathrm{~kg}$ of off-the-shelf, non-permissible, microwave, laboratory, general test equipment were transported to each test location. Two lightweight wideband antennas were mounted on a tripod immediately under a selected test site. Measurements could be made at other test sites in a given location by moving the tripods to those sites. 
Because the equipment required 115 volts ac, and was non-permissible, the test locations available for these measurements were limited.

The equipment used is shown in a block diagram in figure 3. As mentioned earlier, it is not in a form that is suitable for mine measurements, but was adapted for this application for this feasibility study.

The microwave frequency is varied by the sweep oscillator, and the relative, received (reflected) signal amplitude is then shifted down in frequency, detected, and used for the vertical input to an $x-y$ plotter and an oscilloscope. The sweep voltage from the oscillator is used to drive the horizontal input to the plotter and oscilloscope.

The system as shown will not be able to cover the wide range of conditions that occur in normal mining operations.

\subsection{Loveridge Mine}

Measurements were made at two different locations within Loveridge. Initial measurements were made at a location where the coal had been mined approximately ten years ago. Final measurements were made at a location where the coal had been mined approximately two weeks before the measurements were made. It is felt that the results obtained at the latter location should be representative of those obtained at the face during mining operations.

Two points were located in the two-week-old test location where the coal depth changed in a step function. One step was $10.2 \mathrm{~cm}$ high and appeared to be homogeneous coal. The dielectric constant of the coal at that step was measured to be 5.2 . The second step was $15.3 \mathrm{~cm}$ high, and its measured dielectric constant was 4.5 . It should be noted, however, that petrified wood of unknown depth was visible immediately above the microwave antennas at this latter step. The geometry at the second location was such that it was considered impractical to move 
the antennas to avoid the petrified wood. Thus the dielectric constant calculation at the $15.3 \mathrm{~cm}$ step includes the effect of the petrified wood, whose electrical properties are, at this time, unknown. The result in table 1 is an average dielectric constant for the petrified wood-coal mixture at that measurement point. It should also be noted that a tolerance of $\pm 0.6 \mathrm{~cm}$ in the step measurements would cause the dielectric constant bands generated by those tolerances to overlap.

Measurements were made in an area where the estimated thickness of the coal layer was $20-25 \mathrm{~cm}$. This thickness estimate was arrived at by measuring the depth of the nearest drill holes along one side of the tunnel. Due to the curvature of the roof, the depth at the drill hole was greater at the side than that at the center of the haulageway; visual sighting clearly indicated this. Further, actual penetration of the drill bit into the slate was unknown, but was generally thought to be $2.5-5.0 \mathrm{~cm}$.

Using an average of the dielectric constants obtained from the step data, the results given in table I correspond to test points within the general test location.

Antenna rotation experiments were performed to determine if coal exhibited anisotropic polarization effects. The data indicate this effect to be negligible for this particular case.

The dielectric constant of the draw slate was determined by measuring its return loss. This measurement was made at the ten-year-old location by removing the coal under the slate, and measuring the return loss of the slate. The relative dielectric constant for the slate was 63.

\subsection{Bruceton Mine}

The objective in the Bruceton Mine was to prepare coal layers of known thickness and to determine the dielectric constant through measurement. 
Measurements were made at one specially prepared location within the Bruceton Mine. An existing mine face was undercut from the mine floor up toward the draw slate. The undercut extended into the face approximately 1.25 meters so that the antenna-tripod structure could be placed under the test site. The width of the test location was approximately 3.75 meters. The test location was divided into three test areas and the coal layer beneath the draw slate was undercut to depths of 10-13 cm, 20-26 cm, and 39.4-40.6 cm at these three areas. Roof jacks were used to prevent roof fall at these undercut test sites. A picture of the test area is shown in figure 4. Roof jacks, the test areas, antenna-tripod structures and microwave equipment are clearly visible.

The measured dielectric constant obtained in the three prepared coal layers are given in table II.

The measured dielectric constant of the draw slate in the Bruceton mine was 50.3 .

Measured data for these three areas are shown in figures 5, 7, and 9. Calculated data are shown in figures 6, 8, and 10. Although the curves from the measured data are not as smooth as the curves from the calculated data, the basic minima occur at about the same spacing. The calculated curves could be made to agree very closely with the measured curves if enough variation in parameters were used by trial and error; however the significant factor is the spacing between minima. The $40 \mathrm{~cm}$ cases in figures 9 and 10 show the same pattern, except a thinner layer, with deeper nulls (due to less attenuation) spaced further apart, is superimposed on figure 9. Two periodic waves can be extracted from that data. One has a frequency bandwidth of $140 \mathrm{MHz}$ and the other $530 \mathrm{MHz}$. The narrow bandwidth component corresponds to the dielectric constant given in table II for that sample. Substituting the wide bandwidth and the dielectric constant of the sample into (13) results in an 
interface located $10.6 \mathrm{~cm}$ into the coal layer. There are two possible causes for such an interface. First, there could be a dielectric discontinuity at a plane $10.6 \mathrm{~cm}$ above the air-coal interface. Such a discontinuity could be produced by a fracture plane, for example, or a thin layer of petrified wood. Another possibility is that the lower $10.6 \mathrm{~cm}$ of coal has electrical characteristics that are different from those for the upper $29.4 \mathrm{~cm}$ of coal. An on-site visual inspection of this sample was made, and no change in coal texture or color was detected. However, a photographic slide made of that sample shows a change in color of the lower portion of the sample. The lighting for the instant of the photograph was much better than was available from cap lamps. A print of that slide is given in figure 11. The color change for the lower level suggests that the physical characteristics of that test are different, which may also produce a slight change in electrical characteristics and account for the dielectric discontinuity.

\subsection{CONCLUSIONS}

4.1 Microwave techniques can be used to measure the thickness of a layer of coal under most conditions; in case of severe anomalies, the detection of the anomaly may be more important than measuring the layer thickness. The thickness of a coal layer which can be measured this way is at least 0.5 meters.

4.2 The key microwave parameters of all relevant material occurring in the layers must be known under the various conditions that exist in actual mines. This basic information cannot be determined from laboratory data, and must be obtained from in situ measurements, as these parameters are strongly dependent on moisture content, and to some extent, time elapsed since mining. There may be other dependences besides these. The few values measured in this investigation indicate the need for better data. 
4.3 A practical operational system will have to be able to adjust to a rather wide range of electrical properties of materials in order to work under most conditions.

4.4 This limited effort indicates that the frequency range from 1 to $2 \mathrm{GHz}$ may be the best for this application, but until better basic data is available, this is a tentative conclusion.

4.5 Data from the model and analysis developed show general agreement with measured data.

4.6 A11 of the recommendations in the next section are well within the present state-of-the-art.

\subsection{RECOMMENDATIONS}

A substantial program should be undertaken to fully develop and apply this microwave measurement technique. The following factors should be included in such a program in order to achieve a practical, reliable system:

5.1 The electrical characteristics of the various constituents of the layered media must be determined to much better accuracy than they are presently known. This effort should rely mainly on in situ measurements rather than laboratory measurements, and should cover the widely varying conditions that occur normally during mining operations (e.g., varying moisture content). The variations of constituents from one location to another should also be covered (e.g., slate, limestone, etc). Effects of frequency dependence must be considered, probably between $500 \mathrm{MHz}$ and $4 \mathrm{GHz}$.

5.2 The model and analysis given in this report should be used extensively to obtain insights, limits, and allowable uncertainties. It will provide a powerful design tool. The results will be much improved when better data from 5.1 is available. The analysis can also be improved by eliminating assumptions whenever the better data indicates this would be appropriate. 
5.3 Better instrumentation than was used in this feasibility investigation can be developed. Areas for consideration are:

5.3.1 Better horn antennas will give better directivity, sensitivity, etc.

5.3.2 A coaxial directional coupler (better than is presently commercially available) might allow use of a single horn antenna rather than separate transmit and receive antennas.

5.3.3 Data processing and presentation may be improved and/or automated, and a feedback control system added, especially if development of a fully-automated continuous miner is desired.

5.3.4 The system must be permissible, rugged, compact, etc. These considerations will affect the final design.

5.3.5 The system must have enough flexibility and dynamic range to adjust to the rather wide ranges of conditions normally encountered in mining.

5.3.6 Time domain techniques should also be considered. However, instrumentation complexity, cost, and accuracy are the important factors.

\subsection{ACKNOWLEDGMENTS}

Neil Larsen assisted in implementing the analysis; Jocelyn Spencer provided drafting service; Sharon Foote and Janet Becker provided typing service.

None of this investigation would have been possible without the complete cooperation and excellent assistance of: John Burr of Lee Engineering, a Division of Consolidation Coal Company; Paul Carter, Carl Imme1, and Darre1 Auch of Loveridge Mine, of the Mountaineer Division of Consolidation Coal Company; and Mike Pazuchanics and others at the Bruceton Mine of the U.S. Bureau of Mines Mining and Safety Research Center, at Bruceton, Pennsylvania. 


\subsection{REFERENCES}

[1] Ramo, Simon and John R. Whinnery, Fields and Waves in Modern Radio, Second Edition, Chapter 7 (John Wiley \& Sons, Inc., New York and Chapman $\xi$ Hall, Ltd., London, 1953).

[2] Hipp, Jackie E., Soil Electromagnetic Parameters as Functions of Frequency, Soil Density, and Soil Moisture, Proc. of IEEE, Vo1. 62, No. 1, January 1974.

[3] Cook, J.C., Electrical Properties of Bituminous Coal Samples, Geophysics, Vol. 35, No.6, pp.1079-1085, December 1970 . 
APPENDIX

Abbreviations and Symbols

$\mathrm{dBm}$

E

$\mathrm{E}_{\mathrm{O}}$

C

e

f

$\mathrm{s}, \mathrm{l}, \mathrm{d}$

$t$

$\Gamma$

$\alpha$

$\beta$

$\gamma$

$\varepsilon$

$\mu$

$\sigma$

$\omega$

$\mathrm{x}, \mathrm{y}, \mathrm{z}$

$\theta_{i}$

$n_{i}$

$z_{i}$

Signal level with respect to $1 \mathrm{~mW}$, decibels

Electric intensity, $\mathrm{v} / \mathrm{m}$

Maximum electric intensity, $\mathrm{v} / \mathrm{m}$

Velocity of light in free space, $\mathrm{m} / \mathrm{sec}$

Natural logarithm base

Frequency, $\mathrm{H}_{2}$

Thickness of various layers, m

Time, sec.

Reflection coefficient

Attenuation constant, nepers/m

Phase constant, radians/m

Propagation constant

Permittivity, farad $/ m$

Permeability, henry/m

Conductivity, mho/m

Angular velocity, radians/sec

Axes of a rectangular coordinate system

Argument of incident, reflected or refracted waves

in various layers of a medium, radians

Intrinsic impedance of a medium, ohms

Intrinsic impedance of a medium for a wave at oblique incidence, ohms 
Table I. Loveridge mine results.

$$
\varepsilon-4.84
$$

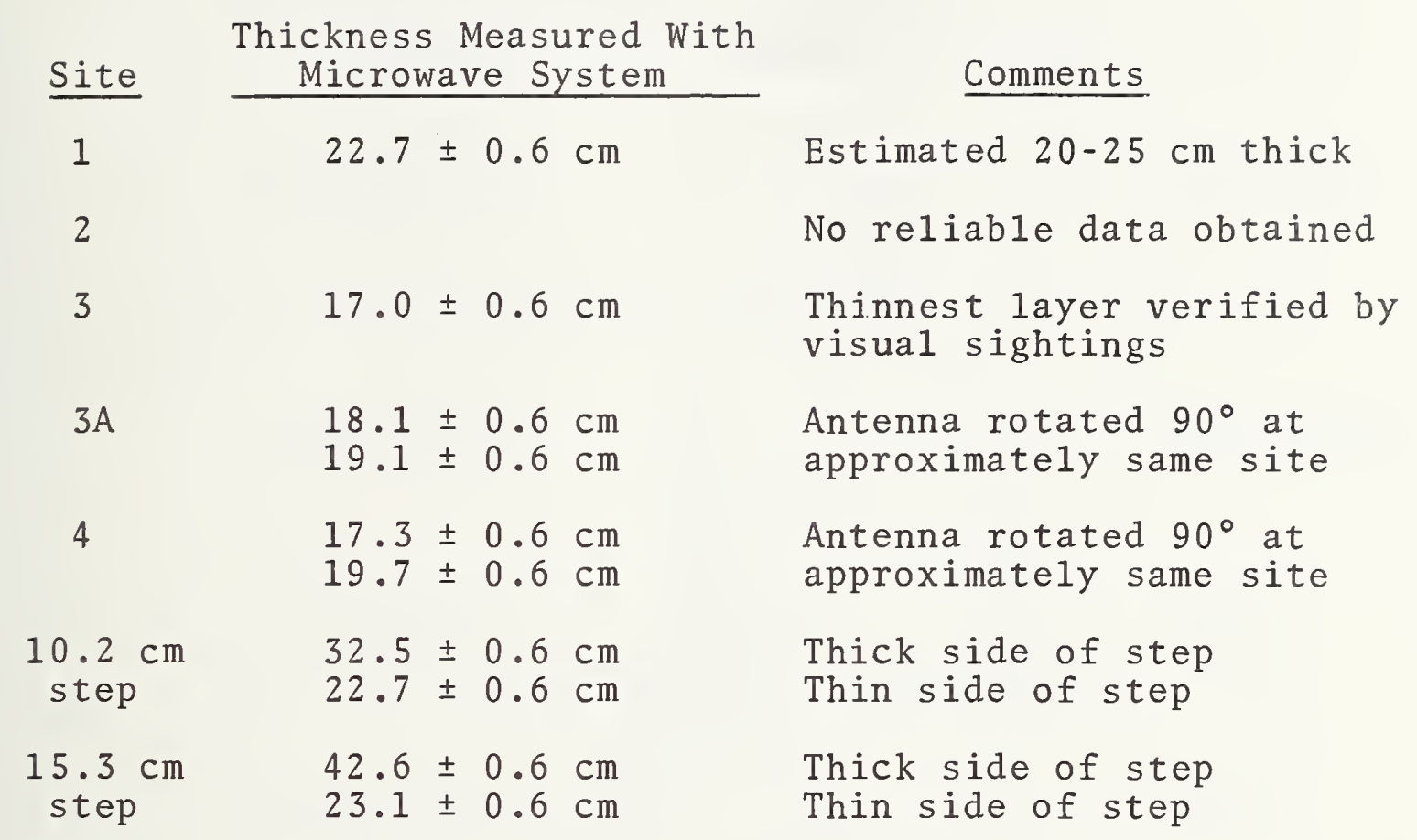


Table II. Bruceton mine results.

\begin{tabular}{|c|c|c|}
\hline Site & $\begin{array}{l}\text { Physical } \\
\text { Thickness }\end{array}$ & $\begin{array}{c}\text { Measured Dielectric } \\
\text { Constant }\end{array}$ \\
\hline 1 & $11.4 \mathrm{~cm}$ & 6.4 \\
\hline 2 & $21.6 \mathrm{~cm}$ & 7.4 \\
\hline 3 & $40.0 \mathrm{~cm}$ & 7.2 \\
\hline
\end{tabular}




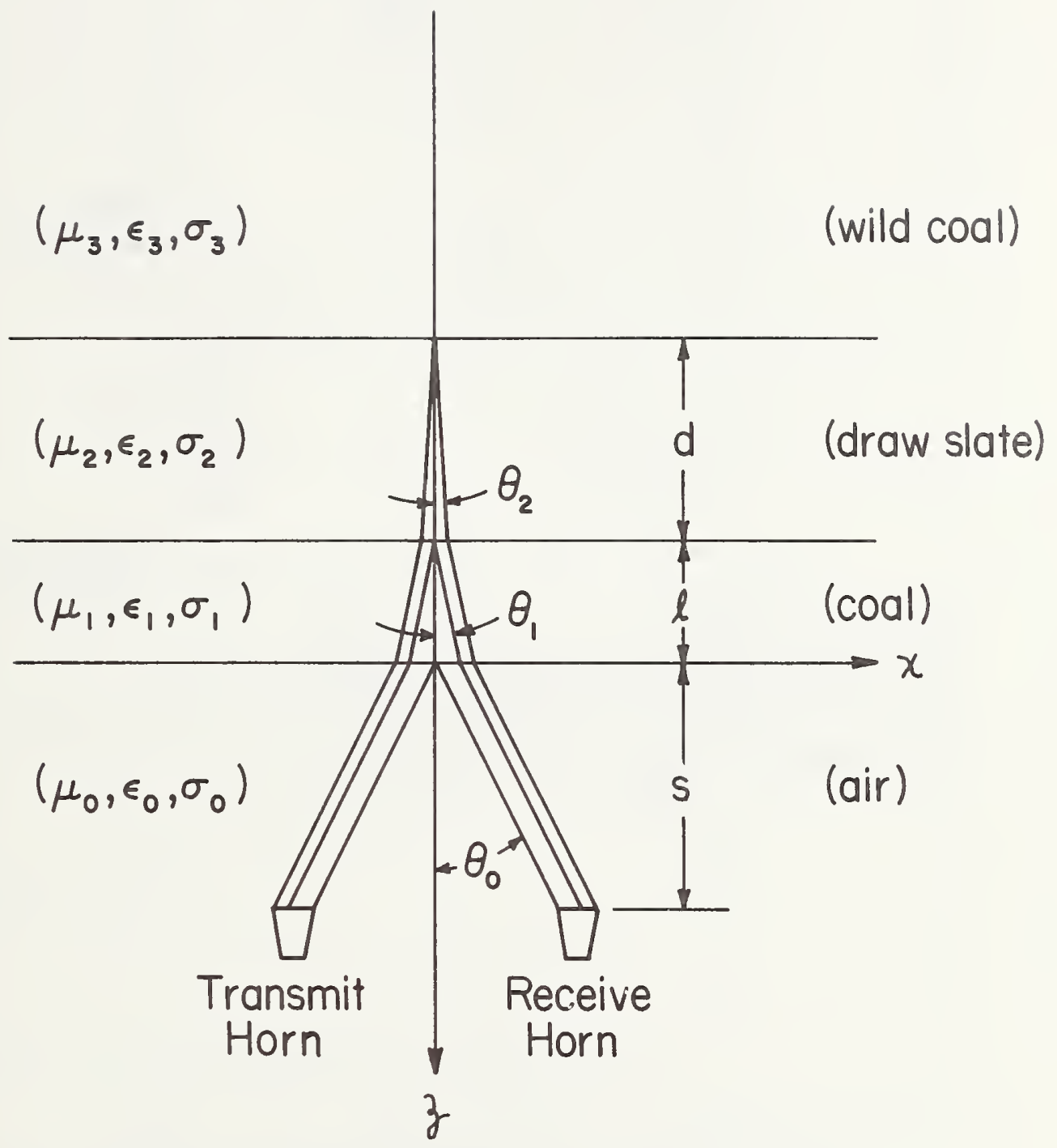

Figure 1. Model used for illustrations and calculations. 


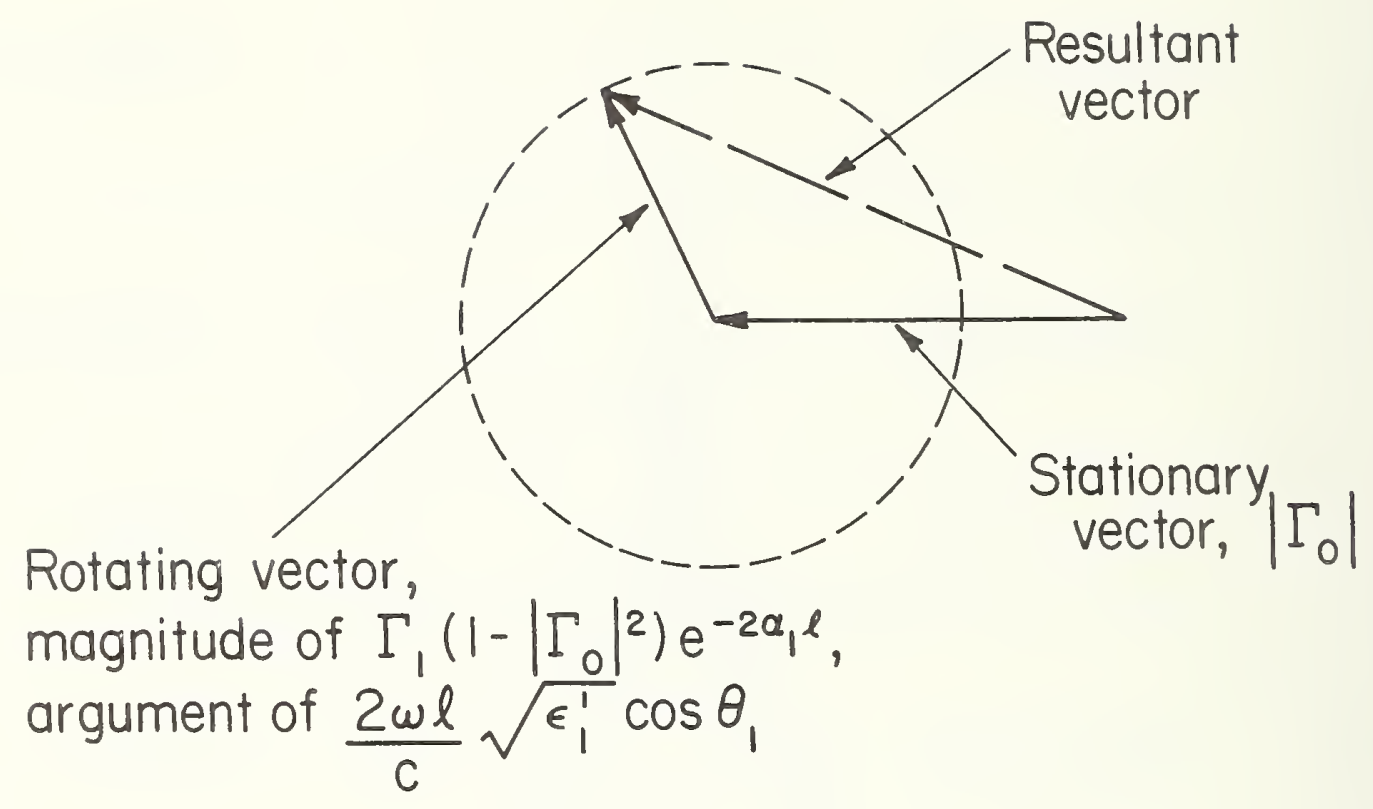

Figure 2. Vector diagram to show derivation of equation 11. 


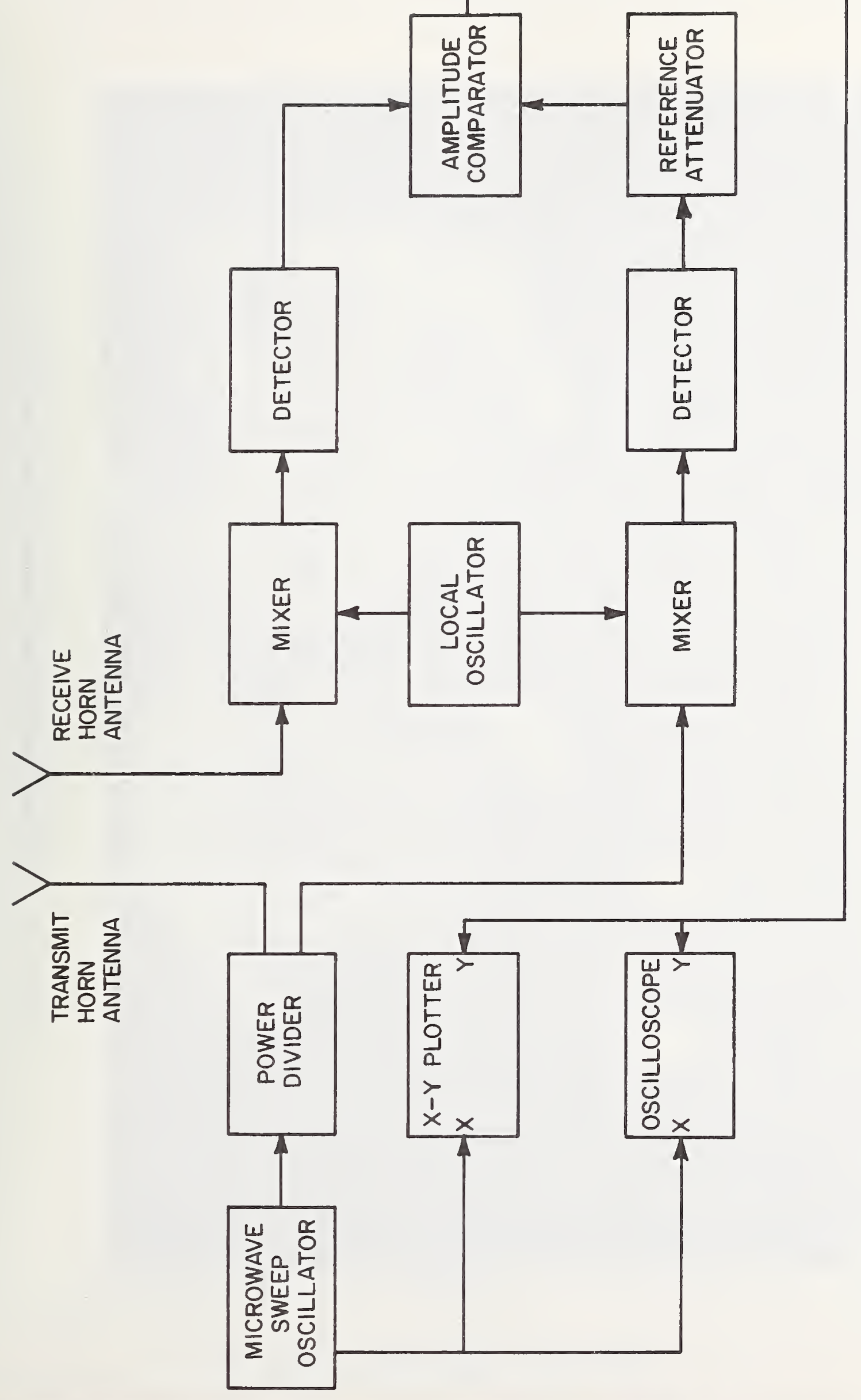

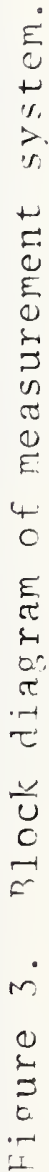




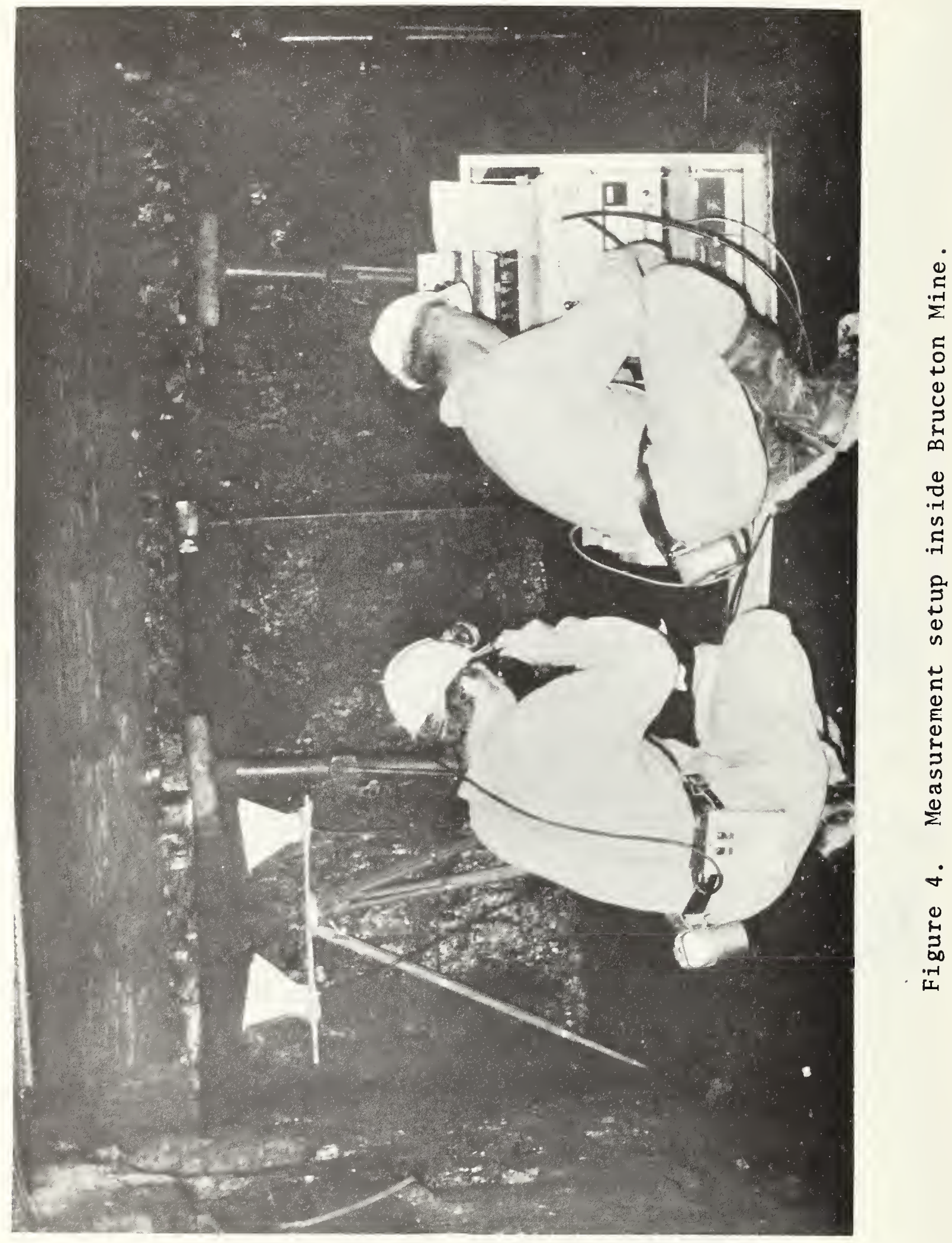




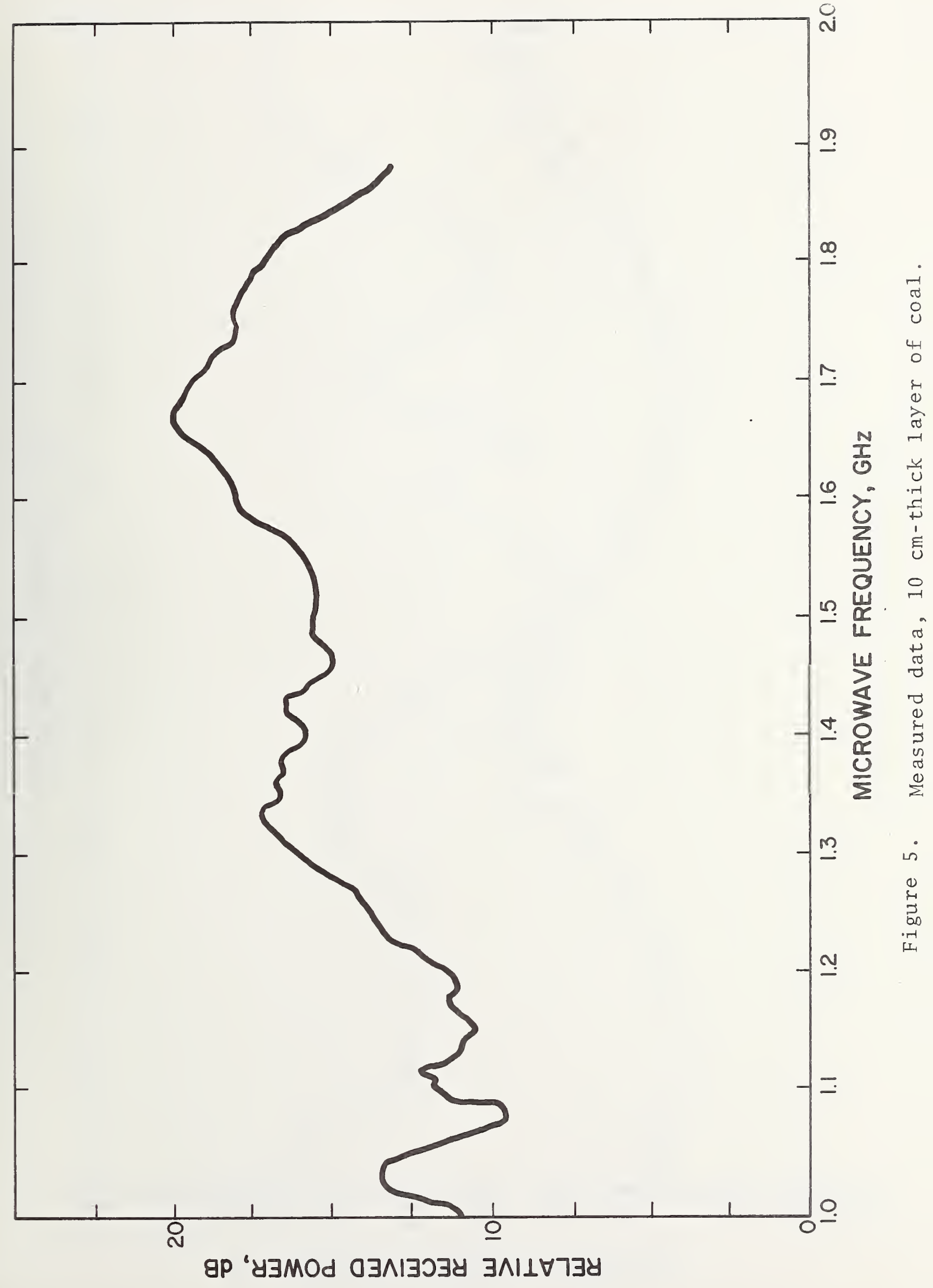




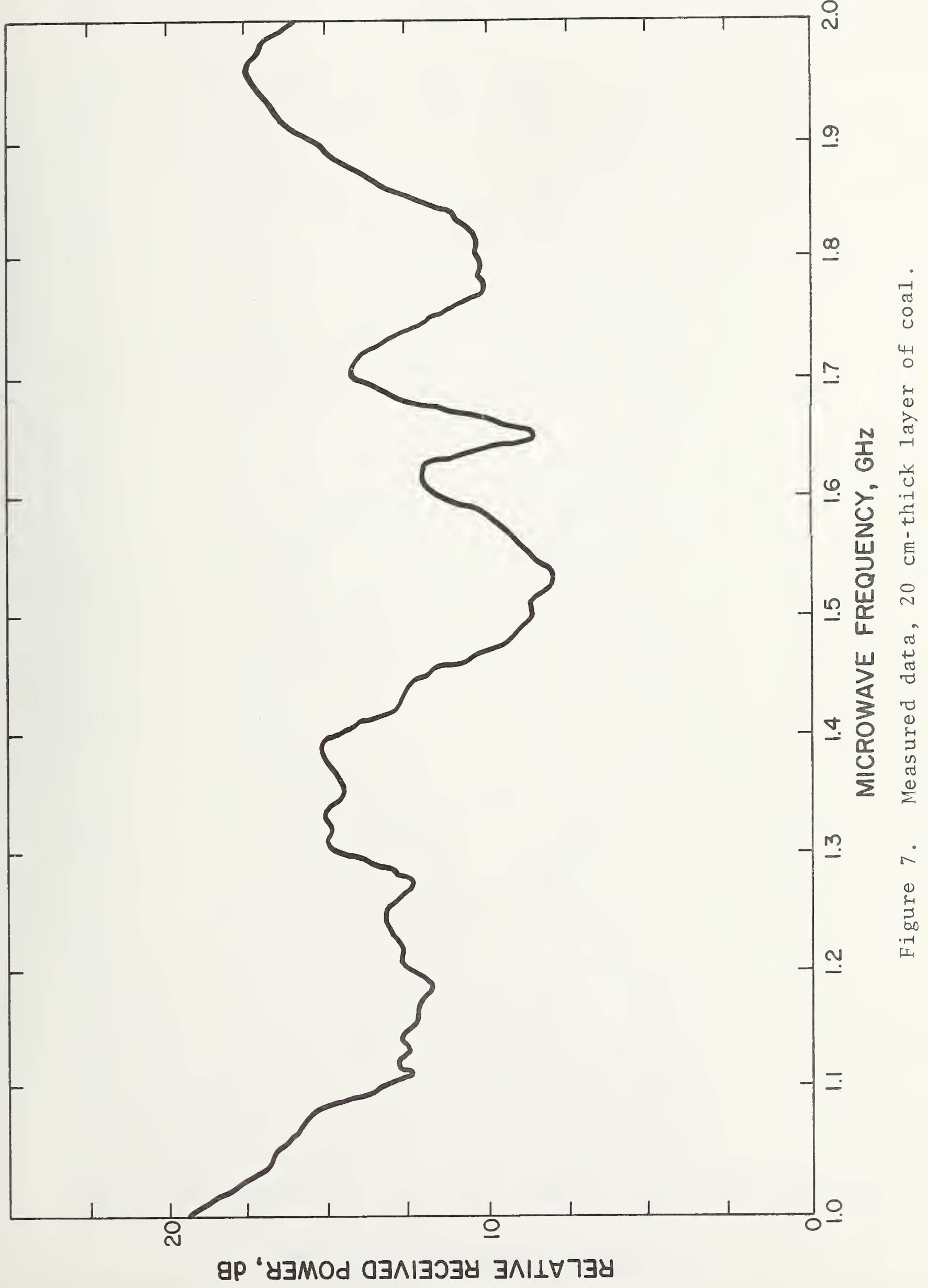




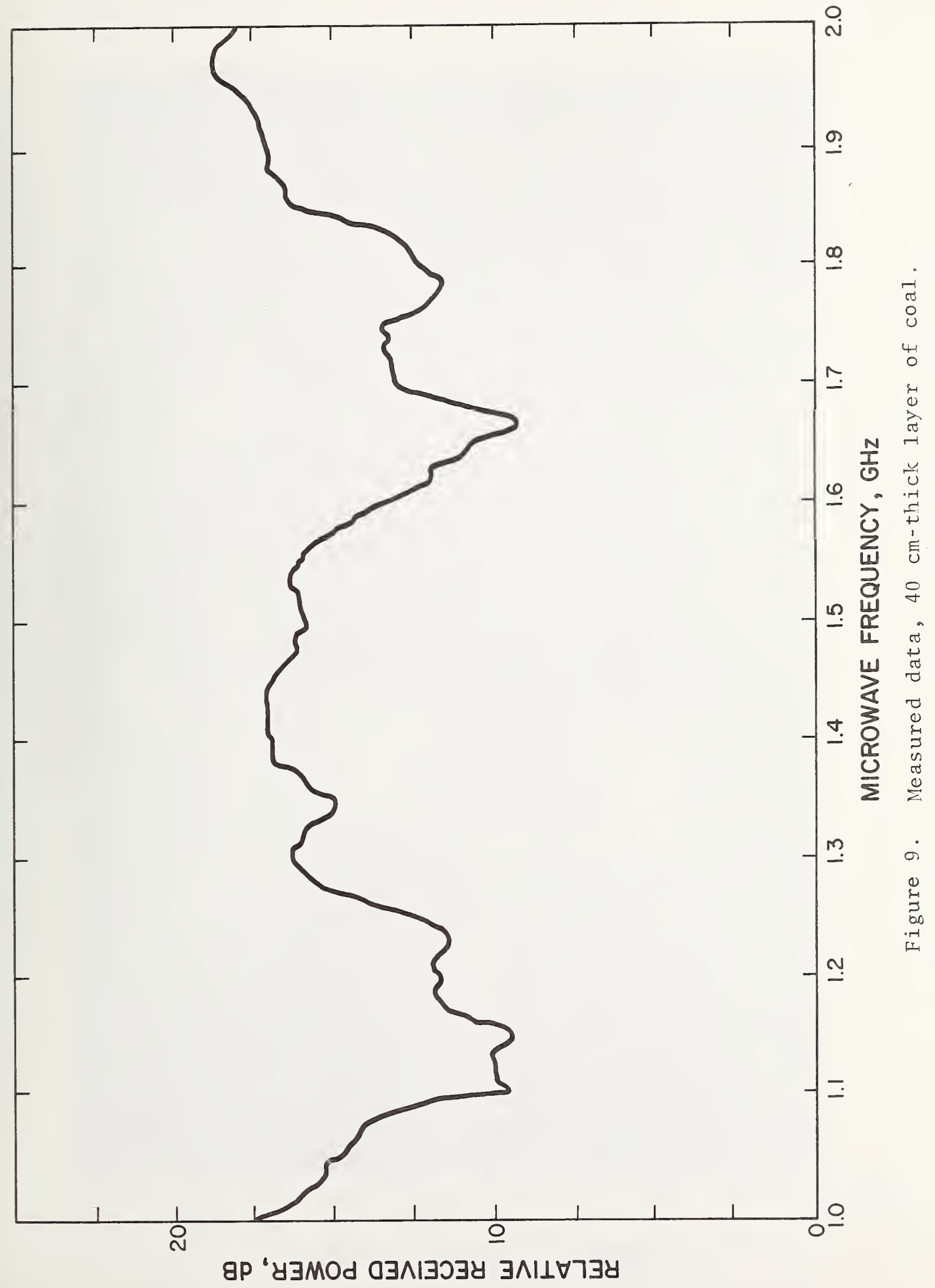




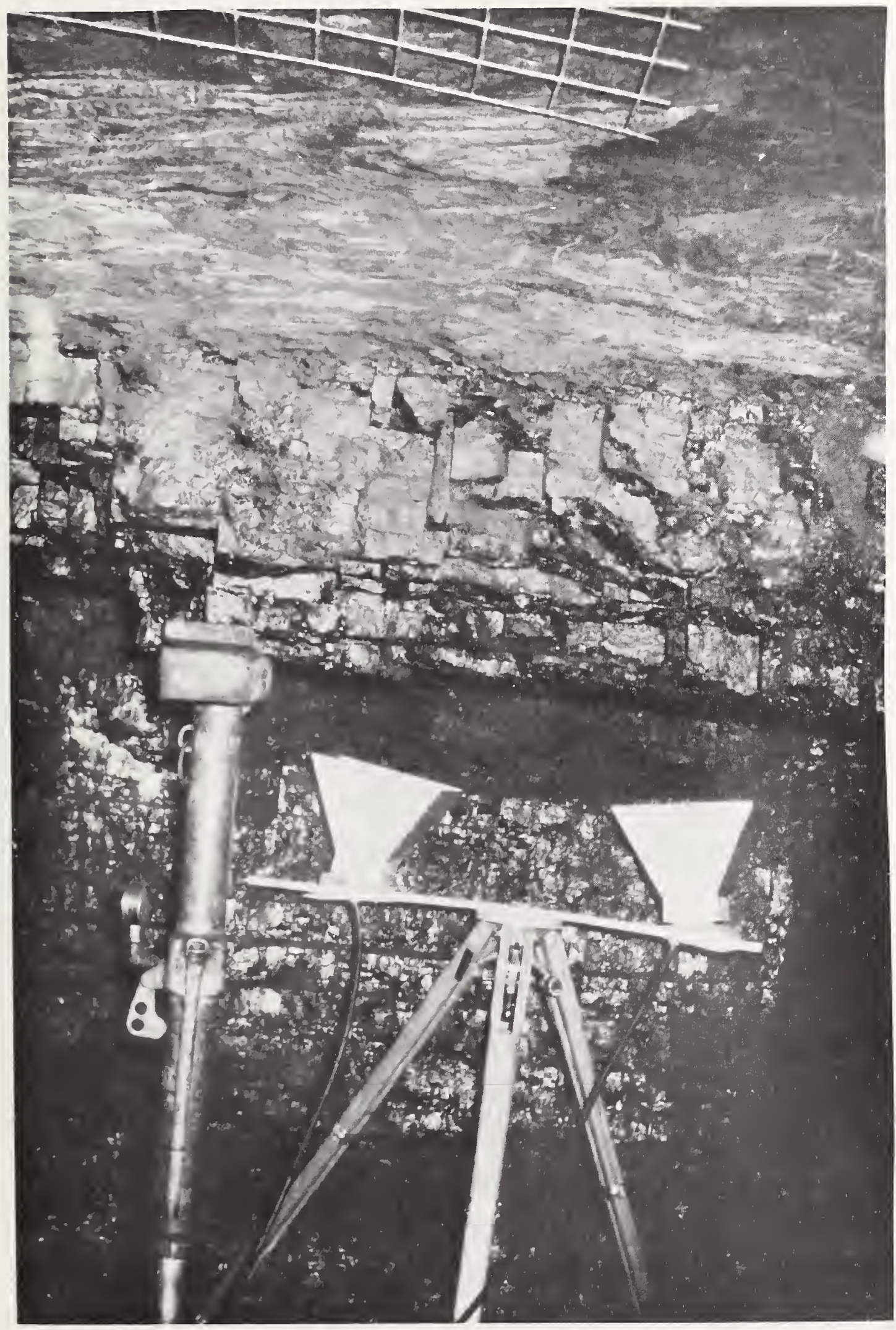

Figure 11. Photo of $40-\mathrm{cm}$ thick layer of coal. 
a 
NBS.114A (REV. 7.73)

\begin{tabular}{|c|c|c|}
\hline $\begin{array}{c}\text { U.S. DEPT. OF COMM. } \\
\text { BIBLIOGRAPHIC DATA } \\
\text { SHEET }\end{array}$ & $\begin{array}{c}\text { 1. PUBLICATION OR REPORT NO. } \\
\text { NBSIR } 74-387\end{array}$ & $\begin{array}{c}\text { 2. Gov't Accession } \\
\text { No. }\end{array}$ \\
\hline
\end{tabular}

4. TITLE AND SUBTITLE

MICROWAVE MEASUREMENT OF COAL LAYER THICKNESS

September 1974

6. Performing Organization Code

7. AUTHOR(S)

Doyle A. Ellerbruch and John W. Adams

9. PERFORMING ORGANIZATION NAME AND ADDRESS

\author{
NATIONAL BUREAU OF STANDARDS, Boulder Labs \\ DEPARTMENT OF COMMERCE \\ WASHINGTON, D.C. 20234
}

12. Sponsoring Organization Name and Complete Address (Street, City, State, ZIP)

U. S. Bureau of Mines

Pittsburgh Mining and Safety Research Center

4800 Forbes Avenue

Pittsburgh, Pennsylvania

15. SUPPLEMENTARY NOTES
8. Performing Organ. Report No. 10. Project/Task/Work Unit No.

2768412

11. Contract/Grant No.

So 144086

13. Type of Report \& Period Covered

$6 / 74-8 / 74$

14. Sponsoring Agency Code

16. ABSTRACT (A 200-word or less factual summary of most significant information. If document includes a significant bibliography or literature survey, mention it here.)

The possibility of using a microwave system to measure coal layer thickness in a mine was investigated. Measurements were made in two different mines near Pittsburgh, $\mathrm{Pa}$., and near Fairview, West Virginia. Frequencies in the range $0.5 \mathrm{GHz}-4.0 \mathrm{GHz}$ were used to measure samples between $10-40 \mathrm{~cm}$ thick. All samples were backed with a naturally-occurring draw slate.

The results indicated a definite possibility of determining layer thickness in most cases, although anomalies may introduce enough error to give misleading results in some cases. Anomaly detection may be very useful in some cases. More experimental data and theoretical study are needed for complete verification.

Data from a model and measured data show considerable agreement. The real value of this model is to point out causes and effects, and what additional information is needed in order to obtain a measurement system that is optimum under most conditions.

The dielectric constant of coal is apparently a function of moisture content. Draw slate seems to have a significantly higher dielectric constant than coal. Also, a layer of coal having different electrical characteristics within the layer was detected with the microwave system.

This technique has great potential if fully developed. It would have significant impact in areas of energy, safety, and productivity.

17. KEY WORDS (six to twelve entries; alphabetical order; capitalize only the first letter of the first key word unless a proper name; separated by semicolons) Automation; coal; coal mine safety; dielectric constant; energy; microwave measurement; nondestructive testing; thickness of coal layer.

18. AVAILABILITY $\mathrm{X}$ Unlimited

For Official Distribution. Do Not Release to NTIS

Order From Sup. of Doc., U.S. Government Printing Office Washington, D.C. 20402, SD Cat. No. C13

Order From National Technical Information Service (NTIS) Springfield, Virginia 22151

\begin{tabular}{|l|c|}
\hline $\begin{array}{l}\text { 19. SECURITY CLASS } \\
\text { (THIS REPURT) } \\
\text { UNCL ASSIFIED }\end{array}$ & 27 \\
\hline $\begin{array}{l}\text { 20. SECURITY CLASS } \\
\text { (THIS PAGE) }\end{array}$ & 22. Price \\
UNCLASSIFIED & USCOMM-DC 29042.P74 \\
\hline
\end{tabular}


